\title{
Bacteraemia after manipulation of the urinary tract. The importance of pre-existing urinary tract disease and compromised host defences $\overline{\vec{*}}$
}

\author{
RICHARD QUINTILIANI \\ M.D. \\ JOSEPH KLIMEK \\ M.D. \\ Burke A. Cunha \\ M.D. \\ Eufronio G. MADERAZo \\ M.D.

\begin{abstract}
Division of Infectious Diseases, Section of Hospital Epidemiology, Department of Medicine and Medical Research Division, Hartford Hospital, Hartford, Connecticut 06115, U.S.A.
\end{abstract}

\begin{abstract}
Summary
The factors related to the occurrence of bacteraemia following urinary tract manipulation were studied in a large community hospital. During a 3-year period, forty-six of 326 episodes of hospital-acquired bacteraemia were associated with urinary tract manipulation. All thirty of forty-six cases felt to be definitely related to urinary tract manipulation (other obvious sources of bacteraemia being absent) had pre-existing urinary tract disease, especially of an obstructive type; only one in this group died from sepsis. The remaining sixteen patients had other possible sources of bacteraemia besides urinary tract manipulation and had disorders associated with defects in host defences; twelve $(75 \%)$ in this group died from overwhelming sepsis. Thus, if bacteraemia occurs in a patient having had urinary tract manipulation but without any underlying urinary tract abnormality or impairment in host defences, its source should be searched for in other areas of the body.
\end{abstract}

\section{Introduction}

Traditionally, most hospital epidemiology programmes have emphasized the distribution of infections but have paid considerably less attention to the determinants. It is becoming increasingly clear that the most important determinant in the acquisition of an infection in hospital is not the environment, but rather the adequacy of the host defence mechanisms of the patient.

This communication deals with the factors in patients in hospital that tend to make urinary tract instrumentation a potentially lethal procedure, as indicated by the development of bacteraemia.

\section{Method}

Since 1970 at Hartford (Connecticut) Hospital, an institution of about 950 beds, epidemiological data have been obtained on all patients who have developed a hospital-associated infection, through the availability of two full-time nurse epidemiologists and a computerized surveillance system.
This surveillance system includes not only a tabusi lation of all patients who have acquired as bacteraemia, bacteriuria, wound infection of pulmonary infection, but also records the responsibles pathogen and the type of medical or surgical serviceo where the infection occurred. The criteria used for the determination of hospital-associated infectionsare those recommended by the Hospital Infections Section of the Center for Disease Control, Atlanta: Georgia (Bennett et al., 1971).

The charts of all patients with hospital-acquired bacteraemias over a 3-year period (October 1970 October 1973) were reviewed for relevant clinicapo and epidemiological data to determine the number:P8 patients in whom the source for this infection was related to infection in the urinary tract. Figures in the tables refer to the strains of organism recoverect from a patient with a hospital-associated infection $\mathbb{D}$ due to this organism (i.e. one per patient per site) $\overrightarrow{\vec{P}}$ If an organism was recovered from more than one site in the same patient, and if this organism was felt to be the cause of the hospital-associated infections at those sites, it would count as more than one case. Thus, one bacterium at two different sites in the same patient producing infection at each of theses sites would be considered as two cases.

The bacteraemia was considered to be definitelys related to instrumentation of the urinary tract onlys if the patient had no other obvious source for bacteraemia other than the urinary tract, and cultures of urine and blood grew the same organismo

A patient was felt to have significant bacteriuria if a single midstream or catheter specimen of urines contained greater than 100000 organisms $/ \mathrm{mln}$ Bladder instrumentation included any invasive procedure involving the urinary tract such as cystoscopy, single straight and indwelling bladder catherization, or recent operation on the urinary tract. Since 1970, sterile, closed, indwelling bladdew catheter systems have been used, except in occasiona ${ }_{0}$ circumstances where three-way catheters witb continuous antibiotic rinses have been employed $\overrightarrow{\mathbb{D}}$ 
TABLE 1. Major organisms associated with hospital-acquired bacteraemia, bacteriuria, and urinary tract (UT) instrumentation (October 1970-October 1973) - number of cases

\begin{tabular}{lccc}
\hline Organism & Bacteriuria & Bacteraemia & $\begin{array}{c}\text { Bacteraemia definitely associated } \\
\text { with UT instrumentation }\end{array}$ \\
\hline Escherichia coli & 1007 & 72 & 9 \\
Proteus sp. & 301 & 11 & 6 \\
Klebsiella pneumoniae & 243 & 29 & 4 \\
Pseudomonas aeruginosa & 296 & 31 & 1 \\
Serratia marcescens & 166 & 8 & 1 \\
Enterococcus spp. & 181 & 20 & 4 \\
Enterobacter sp. & 150 & 23 & 3 \\
Citrobacter sp. & 15 & 2 & 2 \\
Other bacteria & 242 & 130 & 30 \\
Total & 2601 & 326 & 30 \\
\hline
\end{tabular}

During the period of this study, 'open' non-sterile bladder drainage tubes have been prohibited. During the 3-year period of the study there were 29859 indwelling bladder catheters dispensed from the Central Supply Department, and 27375 blood cultures were processed by the Microbiology Laboratory. Serotyping of the organisms was not performed.

\section{Results}

The incidence of bacteriuria induced by urinary tract instrumentation and the number of cases of bacteraemia from all types of hospital-acquired infection are shown in Table 1. Over the period reviewed, there was an average of 867 patients per year with hospital-acquired bacteriuria or an accumulative total of 2601 cases over the 3-year period; during the same time, there were 326 episodes of bacteraemia. However, in only thirty patients $(8 \%)$ was it felt to be definitely related to the urinary tract instrumentation.

TABLE 2. Associated conditions in patients with bacteraemia definitely related to urinary tract (UT) instrumentation

\begin{tabular}{lc}
\hline Condition & No. cases \\
\hline Pre-existing UT disease alone & 23 \\
Pre-existing UT disease and diabetes & 4 \\
Pre-existing UT disease and cirrhosis & 2 \\
Pre-existing UT disease, diabetes mellitus, & 1 \\
cirrhosis & 0 \\
No pre-existing UT disease & 30 \\
Total & \\
\hline
\end{tabular}

Table 2 records the associated conditions in the thirty patients in whom the bacteraemia was definitely related to urinary tract instrumentation. All these patients had pre-existing urinary tract disease. In seven patients with underlying urinary tract disease, there were additional associated illnesses that could further compromise host defences such as diabetes mellitus and Laennec's cirrhosis. Importantly, in no patient was the bacteraemia definitely associated with the instrumentation of the urinary tract unless there was some type of underlying urinary tract disease.

Table 3 records both the type of urinary tract disease and the kind of instrumentation employed in these bacteraemic patients. It is apparent from this chart that the majority of patients not only had obstructive uropathy - usually produced by prostatic disease or calculi - but also underwent more than one type of instrumentation. In fact, eighteen patients had all three types of instrumentation, namely indwelling bladder catheterization, cystoscopy, and operation of the urinary tract. Of the remaining twelve cases, two had both cystoscopy and indwelling bladder catheterization, and ten patients had insertion of a bladder catheter alone. In this group of thirty patients, one patient died from septic shock; this case had obstructive uropathy secondary to cancer of the bladder.

The predominant organism recovered from the blood in these 30 patients was Escherichia coli (9). It was followed in decreasing frequency by Proteus sp. (6), Enterococcus (4), Klebsiella pneumoniae (4), Enterobacter sp. (3), Citrobacter freundii (2) and one case each of Pseudomonas aeruginosa and Serratia marcescens.

\section{Discussion}

Instrumentation of the urinary track has always been a subject of considerable controversy. Although this procedure has been known for some time to be a greater risk in patients with pre-existing renal disease or compromised host defences, quantitation of the actual danger is still unavailable in different types of patients in hospital. The present surveillance data help to fill some of this gap in knowledge regarding the relative hazard of urinary tract instrumentation in a diverse group of patients in a large community hospital.

The present study indicates that urinary tract instrumentation is frequently associated with bacteriuria in all types of patient but is not a common cause of sustained bacteraemia, except for 
TABLE 3. Pre-existing urinary tract disease in patients with bacteraemia definitely related to instrumentation of the urinary tract

\begin{tabular}{|c|c|c|c|c|}
\hline \multirow[b]{2}{*}{ Urinary tract disease } & \multirow[b]{2}{*}{$\begin{array}{l}\text { No. of } \\
\text { patients }\end{array}$} & \multicolumn{3}{|c|}{ Type of instrumentation } \\
\hline & & $\begin{array}{l}\text { Indwelling bladder } \\
\text { catheter }\end{array}$ & Cystoscopy & $\begin{array}{l}\text { Operation } \\
\text { urinary tra }\end{array}$ \\
\hline Calculus ureter & 5 & 5 & 5 & 5 \\
\hline Obstructive uropathy $2^{\circ} \mathrm{BPH}$ & 5 & 5 & 1 & - \\
\hline Postoperative TUR for BPH & 5 & 5 & 5 & 5 \\
\hline Bilateral pyelonephritis & 3 & 3 & - & - \\
\hline Obstructive uropathy $2^{\circ} \mathrm{CA}$ bladder & 3 & 3 & 3 & 2 \\
\hline Bilateral staghorn calculi & 2 & 2 & 3 & 1 \\
\hline Obstructive uropathy $2^{\circ} \mathrm{CA}$ prostate & 2 & 2 & 1 & 1 \\
\hline Atonic bladder, chronic UTI & 1 & 1 & - & - \\
\hline BPH and bladder calculus & 1 & 1 & 1 & 1 \\
\hline Polycystic kidneys & 1 & 1 & - & - \\
\hline Postoperative surprapubic prostatectomy & 1 & 1 & 1 & 1 \\
\hline Right nephrectomy CA kidney & 1 & 1 & 1 & 1 \\
\hline Total & 30 & 30 & 20 & 18 \\
\hline
\end{tabular}

Code: $T U R=$ Transurethral resection. $B P H=$ Benign prostatic hypertrophy. $C A=$ Cancer. $U T I=U$ rinary tract infection.

individuals with pre-existing urinary tract disease. In the past three years, only $8 \%$ of the hospitalassociated bacteraemias could be definitely related to this procedure. This result is surprising in that various reviews (DuPont and Spink, 1969; Freid and Vosti, 1968; Hodgin and Sanford, 1965; Iannini, Claffey and Quintiliani, 1974) on Gram-negative bacteraemia stress the fact that the urinary tract is a common $(37-65 \%)$ portal of entry for these organisms. None of these studies defines the criteria for the establishment of such an association, which may explain the disparity between the present results and those of these other studies.

A surprising result was the complete absence of any sustained bacteraemia definitely associated with bladder catheterization in patients other than those with pre-existing renal disease, usually of an obstructive type and often from prostatic disease. The propensity of bacteraemia to follow manipulation of the prostate was recently shown by Sullivan et al. (1973) in a bacteriological study of 300 patients undergoing four different urological procedures (transurethral resection, cystoscopy, urethral dilatation and urethral catheterization); the highest incidence $(31 \%)$ of bacteraemia occurred in individuals undergoing transurethral resection of the prostate.

Two articles by Martin and Bookrajian (1972) and Martin et al. (1963) are often mentioned as important studies demonstrating the high risk of bacteraemia following indwelling urinary tract catheterization. However, the communication by Martin et al. (1963) basically compares the incidence of bacteraemia in 1960, the last full year when 'open' bladder drainage systems were used, with its frequency in 1962, a year when 'closed' three-way $\vec{O}$ catheter systems, with constant antibiotic rinse of the bladder, were routinely inserted. Although a lessero incidence of Gram-negative bacteraemia was observed in 1962, the total number of patients wi hico bacteraemia was about the same in both years (20900 patients in 1960; 210 patients in 1962). Obvious many other factors besides the type of bladder catheterization, such as different patterns of anti biotic usage, may have produced the reduction ino Gram-negative bacteraemias. Although exact detailso are lacking in Martin et al.'s (1963) article as to how the bacteraemias were definitely related to urinaryo tract manipulation, it is interesting - especially in view of the present findings - that five of seven patients who developed bacteraemia shortly after: three-way bladder catheterization had also under윽 gone concurrently other genito-urinary procedures: (prostatectomy, cystoscopy, cystometry, or urethraB dilatation).

In the other study (Martin and Bookrajian, 1962), eighty-one cases of bacteraemia were evaluated in patients with pyelonephritis; the findings show that forty-four of these patients develope $\frac{}{5}$ bacteraemia within 10 days after the insertion of anindwelling bladder catheter. It is difficult to appraise the results since the definition of pyelonephritis is not provided. Nevertheless, assuming the group of patients had underlying urinary tract disease, the high incidence of bacteraemia following urinarye tract manipulation is still consistent with the presen' observations.

Although additional problems such as diabetes mellitus and Laennec's cirrhosis were found in seven of the patients with pre-existing renal disease 
who developed bacteraemia, their causal relationship, if any, to the bacteraemia is uncertain. Bladder catheterization was performed frequently in elderly individuals, patients with cerebral vascular disease, pregnant women at the time of parturition, postoperative surgical cases, patients with cardiogenic or haemorrhagic shock, but there was not a single case of a definite association between the development of bacteraemia and bacteriuria, unless the patient had pre-existing urinary tract disease.

This observation has important clinical implications since bacteraemia in a patient without obvious urinary tract disease should be considered as having a source from another part of the body. A similar organism may even be isolated both in the blood and urine and still not indicate that the urinary tract is the source for the bacteraemia. Since $E$. coli is the most common pathogen isolated in bacteraemia emanating from biliary or intestinal tract, and since this organism is the most common cause of bacteriuria induced by bladder catheterization, it would be predicted that, following difficult intra-abdominal surgery, $E$. coli could be entirely unrelated to a common source. In such a patient, a normal intravenous pyelogram and serum creatinine should alert the physician to a source other than the urinary tract. Serotyping of the organism would also provide important information on the origin of the bacteraemia; unfortunately, in the present study serotyping was not performed.

Of additional interest was the detection of other organisms in the urine as a frequent cause of bacteriuria but as a rare cause of bacteraemia from a source in the urinary tract. For instance, despite the frequency of bacteriuria from Ps. aeruginosa (296), on only one occasion did this organism produce bacteraemia definitely associated with urinary tract instrumentation.

Although patients with obstructive uropathy appear to be at greatest risk of a bacteraemia from urinary tract instrumentation, most of these individuals survived this complication. Only one patient $(4 \%)$ in this group of cases died from septic shock, and this was a patient with invasive bladder carcinoma and urinary tract obstruction.

It could be argued that transient or even sustained bacteraemia may have occurred in many patients following urinary tract manipulation but it was not detected, because either (a) there was no clinical evidence of sepsis or (b) blood cultures were inadvertently not obtained in febrile patients. Although it is impossible to ascertain the number of times patients did not have blood cultures drawn after the appearance of fever following urinary tract manipulation, it was probably infrequent, since all services at Hartford Hospital have constant house officer coverage and the recommended policy is to obtain two and preferably four blood cultures from any in-patient with an unexplained febrile attack. Supporting this view that blood cultures are commonly obtained is the fact that in the years 1971, 1972 and 1973 there were 7617,10078 and 9680 blood cultures obtained respectively.

It can also be speculated that undetected transient bacteraemias may have clinical relevance in patients with foreign bodies, as artificial cardiac valves or arterial grafts in contact with the circulatory system, with underlying valvular or congenital heart disease, or with severely compromised host defences, since in these settings even brief exposure to an organism may result in the establishment of an infectious disease process. Thus, it must be emphasized that in these types of patient, even without intrinsic or obstructive urinary tract disease, urinary tract manipulation may still be a hazardous procedure.

From this epidemiological survey, sustained bacteraemia was found to be definitely related to urinary tract instrumentation only in patients with underlying urinary tract disease, usually of an obstructive nature, and was greatest in those who underwent multiple urinary tract manipulation. In bacteraemia patients with indwelling bladder catheters but without concomitant urinary tract disease, a careful search should be made for another source of the bacteraemia.

\section{References}

Bennett, J.V., Garner, J.S., Scheckler, W.E., Maki, D.G. \& BRACKMAN, P.S. (1971) Surveillance of nosocomial infections. In: Proceedings; International Conference on Nosocomial Infections, p. 277. Center for Disease Control, August 3-6, 1970. Chicago, American Hospital Association.

DuPoNT, H.L. \& SPINK, W.W. (1969) Infections due to Gram-negative organisms: an analysis of 860 patients with bacteremia at the University of Minnesota Medical Center, 1958-1966. Medicine, 48, 307.

FreID, M.A. \& Vosti, K.L. (1968) The importance of underlying disease in patients with Gram-negative bacteremia. Archives of Internal Medicine, 121, 418.

HodgIN, U.G., \& SANFORd, J.P. (1965) Gram-negative rod bacteremia: an analysis of 100 patients. American Journal of Medicine, 39, 952.

IANNini, P.B., Claffey, T. \& Quintiliani, R. (1974) Bacteremic Pseudomonas pneumonia. Journal of the American Medical Association, 230, 558.

Maiztegui, J.I., Biegeleisen, J.Z., Cherry, W.B., \& Kass, E.H. (1965) Bacteremia due to Gram-negative rods: a clinical, bacteriologic, serologic and immunofluorescent study. New England Journal of Medicine, 272, 222.

Martin, C.M., \& Bookrajian, E.N. (1962) Bacteriuria prevention after indwelling urinary catheterization. Archives of Internal Medicine, 110, 703.

Martin, C.M., Vaquer, F., Meyers, M.S. \& El-Dadah, H. (1963) Prevention of Gram-negative and bacteremia associated with indwelling urinary-tract catheterization. Antimicrobial Agents and Chemotherapy, 3, 617.

Sullivan, N.M., SutTer, V.L., Mims, M.M., Marsh, V.H. \& FeINGold, S.H. (1973) Clinical aspects of bacteremia after manipulation of the genitourinary tract. Journal $o$, Infectious Diseases, 127, 49. 